\title{
Associations between Different Diagnostic Approaches for Child and Adolescent Psychopathology
}

\author{
Marianne C. Kasius and Robert F. Ferdinand \\ Erasmus University and Sophia Children's Hospital, Rotterdam, The Netherlands \\ Henk van den Berg \\ RIAGG RNO, Rotterdam, The Netherlands \\ Frank C. Verhulst \\ Erasmus University and Sophia Children's Hospital, Rotterdam, The Netherlands
}

\begin{abstract}
Objective: To test the convergence between the empirical-quantitative approach of the Child Behavior Checklist (CBCL) and the clinical-diagnostic approach of the DSM. Method: The parent version of the NIMH Diagnostic Interview Schedule for Children (DISC), version 2.3 , was administered after completion of the CBCL for 231 children and adolescents consecutively referred to an outpatient mental health clinic. Results: Of the subjects with a DSM-III-R diagnosis, $60 \%$ scored in the clinical range of the CBCL total problem score. The Withdrawn scale predicted affective and anxiety disorders. The Somatic Complaints scale predicted anxiety and mood disorders and Attention Deficit Hyperactivity Disorder. The Anxious/Depressed scale predicted anxiety and mood disorders and, to a lesser extent, disruptive behavior disorders. The Social Problems scale predicted Oppositional Defiant Disorder. The Attention Problems scale was the only significant predictor of "pure" Attention Deficit Hyperactivity Disorder (ADHD). The Aggressive Behavior scale predicted several disruptive behavior disorders, and Major Depression. The Delinquent Behavior scale was strongly associated with Conduct Disorder. Conclusions: Empirically based CBCL scale scores and DISC-P based DSM-III-R diagnoses converged. However, both approaches do not converge to a degree that one approach can replace the other. Instead, combining both approaches may be valuable by adding information from one approach that is not captured by the other.
\end{abstract}

Keywords: Assessment, classification, diagnosis, Child Behavior Checklist, Diagnostic Interview Schedule.

Abbreviations: ADDH: Attention Deficit Disorder with Hyperactivity; ADHD: Attention Deficit Hyperactivity Disorder; CBCL: Child Behavior Checklist; DISC: Diagnostic Interview Schedule for Children.

\section{Introduction}

Two main approaches to assess child and adolescent psychopathology can be distinguished: the clinicaldiagnostic and the empirical-quantitative approaches. Traditional clinical-diagnostic systems such as the Diagnostic and Statistical Manual of Mental Disorder (American Psychiatric Association, 1980, 1987, 1994) employ criteria for disorders that are the result of consensus through negotiations among panels of experts. This approach can be characterized as working from the

Requests for reprints to: F.C. Verhulst, Erasmus University/Sophia Children's Hospital, PO Box 2060, 3000 CB Rotterdam, The Netherlands (Email: verhulst@psys.azr.nl). "top down", because it starts from decisions about which disorders should be included and about the criteria for defining such disorders (Verhulst \& Achenbach, 1995). In this way, disorders reflect current clinical practice and are defined by a rather arbitrary set of criteria. The assessment strategy that is typically followed to derive DSM diagnoses is the clinical interview, either unstandardized or in a standardized form.

In contrast, the empirical-quantitative approach, which is derived from psychometric concepts, uses quantitative procedures to determine empirically which characteristics tend to co-occur to form syndromes. The empirical approach works from the "ground up", because it starts with large numbers of items that describe a broad range of behavioral/emotional problems that are scored for large samples of subjects. Multivariate stat- 
istical procedures are used to identify which problem items go together to form syndromes.

The assessment strategy that is typically followed to obtain quantitative scores for the empirically derived syndromes uses standardized rating scales. Rating scales have the advantage that normative data can readily be made available, and that decisions on the number and severity of problems that should be regarded as deviant are based on actual distributions of scores in populations rather than on an arbitrary number of criteria.

The mere fact that the two approaches as described here exist together shows that neither of them fully satisfies the numerous questions with regard to assessment and diagnosis of child and adolescent psychopathology. The combination of both diagnostic paradigms may be valuable if they compensate for the weaknesses and augment the strengths for each approach.

There are few studies that have empirically tested the relationship between the two approaches. In order to test the convergence between the two diagnostic paradigms, it is important that the information which is compared is derived from the same type of informant. For example, if we compare the parents' Child Behavior Checklist (CBCL) ratings with DSM diagnoses derived from clinical interviews with the child, we do not know if discrepancies reflect the difference in diagnostic approaches or the use of different informants (parent versus child). However, if we compare parents' CBCL ratings with results from diagnostic parent interviews, we are able to test the method variance, because we keep the source of information constant. Of course, even if we use the same type of informant, there is measurement error. In our example, if parents complete the $\mathrm{CBCL}$ and then have a clinical interview some time later, we have to take account of test-retest unreliability. Most studies testing the relationship between the CBCL and DSM diagnoses have used parents as informants.

Edelbrock and Costello (1988) were the first to publish relationships between DSM-III diagnoses derived from one of the earliest versions of the parent DISC (NIMH Diagnostic Interview Schedule for Children), and the pre1991 CBCL scales (Achenbach \& Edelbrock, 1983) that preceded the current version (Achenbach, 1991b). There was considerable overlap between CBCL scale scores and DISC diagnoses in their sample of 270 clinically referred children aged 6 to 16 years. The strongest relations were found between the CBCL scales Hyperactive, Delinquent, and Depressed with DISC-derived diagnoses of Attention Deficit Disorder, Conduct Disorder, and Depression/Dysthymia, respectively.

A problem with clinical samples, such as the one used in the study by Edelbrock and Costello (1988), is that they may be selected and contain disproportionally high levels of subjects with multiple problems. However, a problem with nonclinical samples is that very large samples are needed to find enough cases with relatively infrequent diagnoses. Jensen, Salzberg, Richters, and Watanabe (1993) studied the relationship between the CBCL and the DISC 2.1 (Diagnostic Interview Schedule for Children; NIMH, 1992) in 201 5- to 17-year-olds from military families. In this sample, correlations between DSM-III-R diagnoses and the pre-1991 CBCL scale scores were computed for ADHD and anxiety disorders, but not for other disorders due to the low prevalence in this sample, which may not be representative for children outside of military settings.

Gould, Bird, and Staghezza Jamarillo (1993) studied the relationship between the pre-1991 CBCL scales and DISC-derived DSM-III diagnoses in a community sample of 308 children aged 6 to 16 from Puerto Rico. The authors reported moderate convergence between both approaches, although a number of DSM-III diagnoses needed to be combined, or could not be analyzed due to the low prevalence of these disorders in the sample.

The poor differentiation in the relationship between both approaches may be a result of the high rate of comorbidity that is intrinsic to child and adolescent psychopathology. Steingard, Biederman, Doyle, and Sprich-Buckminster (1992) compared pre-1991 CBCL scales with DSM-III diagnosis of Attention Deficit Disorder with Hyperactivity (ADDH) with and without comorbid diagnoses in 6- to 16-year-old boys participating in family genetic studies. They found that subjects with ADDH scored significantly higher on all CBCL scales. However, it was the subgroup of boys with ADDH in combination with other diagnoses that accounted for these results. Similarly, Biederman et al. (1993), who studied 133 clinically referred 6- to 17-yearolds and 118 nonreferred children, found that subjects with a DSM-III-R diagnosis of Attention Deficit Hyperactivity Disorder (ADHD), and at least one other DSM-III-R diagnosis, had significantly higher scores on all CBCL scales than children without comorbidity.

The studies discussed so far all used versions of the CBCL scales and/or DISC that have now been replaced by newer versions. Achenbach (1991b) has constructed scales that are similar across both sexes, age groups 6 to 11 and 12 to 18 , and with parents, teachers, and youths as informants. The earlier versions of the scales that were used in most studies described above were different across sexes, age groups, and informants. Also the DISC has been revised over the years to improve its psychometric properties and to match the newer editions of DSM. The majority of studies described above used DISC versions that matched DSM-III criteria.

The purpose of the present study was to test the convergence between the newest (1991) version of the CBCL scales with DSM-III-R diagnoses derived from the parent version of the DISC 2.3 in a sample of 231 6- to 16-year-old clinically referred children and adolescents.

\section{Method}

\section{Procedure}

Parents of 325 consecutive patients aged 6 to 16 years who were referred to one outpatient unit for child and adolescent psychiatry were requested by mail to participate in the study, which was especially designed to test the convergence of the two diagnostic paradigms. Parents who could not be contacted by phone to make an appointment for the interview were visited at home to make an appointment.

Parents of 148 boys (mean age $=10.4$ years), and 83 girls (mean age $=10.6$ years) cooperated (response $=71 \%$ ). Information was provided by mothers $(87 \%)$, or fathers or parent 
Table 1

Frequencies of DSM-III-R Diagnoses and of Subjects Scoring in the Clinical Range of CBCL Syndrome Scales $(\mathrm{N}=231)$

\begin{tabular}{|c|c|c|c|}
\hline \multicolumn{4}{|c|}{ DISC/DSM-III-R Diagnoses } \\
\hline Simple Phobia & $44(19 \%)$ & Any Tic Disorder & $19(8 \%)$ \\
\hline Social Phobia & $12(5 \%)$ & Major Depression & $24(10 \%)$ \\
\hline Agoraphobia & $7(3 \%)$ & Dysthymia & $27(12 \%)$ \\
\hline Panic Disorder & $2(1 \%)$ & Mania & $7(3 \%)$ \\
\hline Separation Anxiety & $17(7 \%)$ & Hypomania & $1(-\%)$ \\
\hline Avoidant Disorder & $7(3 \%)$ & Any Mood Disorder & $42(18 \%)$ \\
\hline Overanxious Disorder & $16(7 \%)$ & Psychosis screen & $12(5 \%)$ \\
\hline Generalized Anxiety Disorder & $15(7 \%)$ & $\begin{array}{l}\text { Attention-deficit Hyperactivity } \\
\text { Disorder }\end{array}$ & $70(30 \%)$ \\
\hline Obsessive Compulsive Disorder & $1(-\%)$ & Oppositional Defiant Disorder & $33(14 \%)$ \\
\hline Any Anxiety Disorder & $79(34 \%)$ & Conduct Disorder & $13(6 \%)$ \\
\hline Bulimia/Anorexia & - & Any Disruptive Behavior Disorder & $86(37 \%)$ \\
\hline Enuresis & $21(9 \%)$ & Any Substance Use Disorder & $2(1 \%)$ \\
\hline Encopresis & $6(3 \%)$ & Any Disorder & $146(63 \%)$ \\
\hline \multicolumn{4}{|c|}{ CBCL Scores in the Clinical Range } \\
\hline Withdrawn & $21(9 \%)$ & Delinquent Behavior & $29(13 \%)$ \\
\hline Somatic Complaints & $20(9 \%)$ & Aggressive Behavior & $49(21 \%)$ \\
\hline Anxious/Depressed & $36(16 \%)$ & Internalizing & $90(39 \%)$ \\
\hline Social Problems & $44(19 \%)$ & Externalizing & $92(40 \%)$ \\
\hline Thought Problems & $12(5 \%)$ & Total Problem score & $109(47 \%)$ \\
\hline Attention Problems & $50(22 \%)$ & & \\
\hline
\end{tabular}

substitutes (13\%). According to the occupational level of the parents, the socioeconomic status (SES) was assessed, with scores of 1 or $2=$ low $(N=32 \%), 3$ or $4=$ medium $(N=$ $27 \%$ ), and 5 or $6=$ high $(N=41 \%)$ SES (Van Westerlaak, Kropman, \& Collaris, 1975).

Parents were interviewed with the parent version of the DISC, by interviewers who were trained by two of the authors (RF and MK) who, in turn, were trained at the Columbia University Training Centre in New York.

In the week prior to the interview, the parent who was to be interviewed completed the CBCL. The interviewer was not acquainted with the CBCL scores.

\section{Instruments}

The Child Behavior Checklist. The CBCL (Achenbach, 1991b) was used to obtain standardized parents' reports of children's problem behaviors based on the preceding 6 months. The good reliability and validity established by Achenbach (1991b) were confirmed for the Dutch translation (Verhulst, Berden, \& Sanders-Wondstra, 1985).

Achenbach (1991a) constructed the following eight syndrome scales: Withdrawn, Somatic Complaints, and Anxious/ Depressed (constituting the Internalizing group), Delinquent Behavior and Aggressive Behavior (constituting the Externalizing group), and Thought Problems, Social Problems and Attention Problems.

For the syndrome scales, a $T$ score above 70 , and for the total problem score a $T$ score above 63 represent the clinical range (Achenbach, 1991a). These thresholds represent scores that discriminated well between referred and nonreferred subjects. However, these thresholds are rather arbitrary and can be adjusted to improve discrimination in some samples.

The NIMH Diagnostic Interview Schedule for Children. The DISC, version 2.3, is a highly structured interview to assess the more common DSM-III-R diagnoses (American Psychiatric Association, 1987) found in children and adolescents. Unless otherwise specified, the timeframe of the DISC is the past 6 months. The DISC 2.3 covers the diagnostic categories listed in Table 1.

The DISC has two parallel forms: DISC-C administered directly to the child or adolescent, and DISC-P administered to the parent or parent substitute. The DISC-P was used in the present study. The reliability and validity of the earlier versions of the DISC 2.3, the DISC-R and the DISC 2.1, were supported by Piacentini et al. (1993), Schwab-Stone et al. (1993), and Shaffer et al. (1993). The thresholds above which a subject satisfies the criteria for a DSM diagnosis are rather arbitrary. Diagnostic thresholds and diagnostic content vary across the different editions of DSM.

\section{Results}

\section{Frequencies of CBCL Scores and DSM-III-R Diagnoses}

Frequencies of CBCL syndrome scores in the clinical range $(>T 70)$ and DISC/DSM-III-R diagnoses are presented in Table 1.

\section{Associations between CBCL Scales and DSM-III-R Diagnoses}

Eighty-six of the 231 subjects ( $37 \%$ ) had a combination of a CBCL total problem score in the clinical range ( $>T 63$ ) and at least one DSM-III-R diagnosis (sensitivity $=59 \%$, specificity $=73 \%$ ).

To test the ability of CBCL syndrome scale scores to predict DSM-III-R diagnoses, we performed logistic regression analyses. Analyses for specific DSM-III-R diagnoses as dependent variables were conducted for disorders with a prevalence $\geqslant 5 \%(N=12)$. Diagnostic groups that did not exceed a frequency of $5 \%(N=12)$ were not analyzed because of lack of statistical power. DSM-III-R diagnoses were entered in the regression analyses as $1=$ present and $0=$ no diagnosis present. In this way, diagnosed subjects were compared with 
M. C. KASIUS et al.

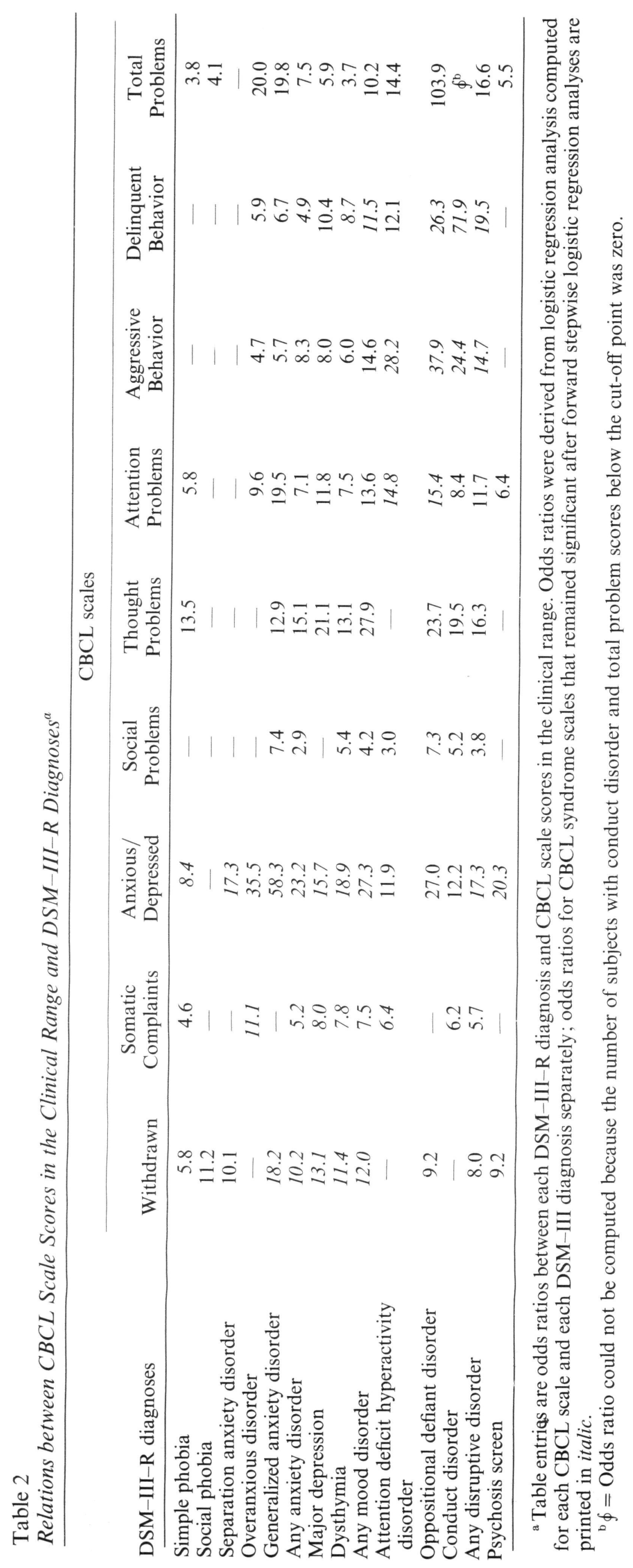


"controls" who did not fulfil the criteria for any DSM-III-R diagnosis (although they were subjects who were referred and cannot be equated with nonreferred subjects).

In addition, we tested associations between the CBCL scale and the following broad diagnostic groups in which DSM-III-R diagnoses were collapsed: any anxiety disorder (all nine anxiety disorders listed in Table 1 combined), any mood disorder (Major Depression, Dysthymia, Hypomania, and Mania), and any disruptive behavior disorder (Oppositional Defiant Disorder, Conduct Disorder, and Attention Deficit Hyperactivity Disorder).

In a first set of separate logistic regression analyses, each CBCL syndrome and total problem score, sex $(0=$ boy, $1=$ girl $)$, and age $(0=6-11$ years, $1=12-16$ years $)$ were entered in each analysis. CBCL syndrome scores were recoded as $0(\leqslant T 70)$ and $1(>T 70)$. CBCL total problem score was recoded as $0(\leqslant T 63)$ and 1 ( $>T 63)$. Table 2 shows the results of these analyses.

A second set of regression analyses was performed to test which $\mathrm{CBCL}$ syndrome scales predicted a DSM-III-R diagnosis independently of other CBCL scales. We used forward stepwise regression analyses with CBCL scores that predicted a DSM-III-R diagnosis in the first set of analyses, with age and sex as possible predictor variables (criterion for entrance: $p<.05$; criterion for removal $p=.1$; likelihood ratio test). Odds ratios that remained significant after this stepwise procedure, in which the effect of one scale was assessed with the effects of other scales partialled out, are printed in italic in Table 2.

The Wald statistic was used to test the significance ( $p$ $<.05$ ) of independent variables, whereas model $\chi^{2}$ tests $(d f=3)$, were used to test the significance $(p<.05)$ of full regression models. The number of tests in this study may suggest the need for a correction for chance findings for multiple tests, such as a Bonferroni procedure. However, the probability of chance findings in the logistic regression analyses was minimized by applying significance tests for the full regression models.

\section{The Role of Comorbidity}

So far, we have analyzed the ability of CBCL scales to predict the presence of a DSM-III-R diagnosis versus the absence of any diagnosis, irrespective of the presence of other DSM-III-R diagnoses for the same subject. Of the 146 subjects who fulfilled criteria for a DSM-III-R diagnosis, 53 had one, and 93 subjects had more than one, diagnosis. To assess the effect of comorbidity among DSM-III-R disorders, subjects who fulfilled criteria for each of the diagnostic groups any anxiety disorder, any mood disorder, any disruptive disorder, or ADHD were divided into subjects who did and subjects who did not fulfil criteria for any other diagnosis. In this way we were able to assess the ability of CBCL scales to predict the presence of "pure" DSM-III-R diagnoses. Diagnoses of specific disorders other than ADHD did not contain the minimum of 10 "pure" cases that we set arbitrarily. For these diagnoses, subjects could not be divided into those with and without comorbidity.

None of the CBCL scales (including total problem score) significantly predicted the "pure" categories of any anxiety disorder and any mood disorder. "Pure" any disruptive disorder was predicted by the CBCL scales Social Problems (odds ratio 6.5), Attention Problems (17.1), Aggressive Behavior (4.2), and Total Problems (9.7). In the stepwise logistic regression analysis of the CBCL syndrome scales, only Social Problems and Attention Problems independently predicted the presence of "pure" any disruptive disorder.

"Pure" ADHD was significantly predicted by the CBCL scales Social Problems (odds ratio 5.5), Attention Problems (24.0), and Total Problems (6.9), with Attention Problems as the only independent predictor in the stepwise regression analysis.

\section{Differences in Mean CBCL Scale Score for Broad $D S M-I I I-R$ Categories}

To test differences in mean CBCL scale scores between three combined broad groups of DSM-III-R diagnoses, we performed one-way ANOVAs $(d f=2)$ to compare mean CBCL scale scores between anxiety disorders $(N=$ $27)$, mood disorders $(N=12)$, and disruptive disorders $(N=40)$ with post hoc Student Newman-Keuls tests. For each of the three categories, disorders comorbid to another category were excluded from analysis.

We found significantly higher mean scores for anxiety disorders versus disruptive disorders on the $\mathrm{CBCL}$ Withdrawn scale $(F=3.6, p<.05)$; higher scores for disruptive disorders versus mood disorder on the $\mathrm{CBCL}$ Social Problems $(F=3.2, p<.05)$ and Aggressive Behavior scales $(F=7.4, p<.01)$; and higher scores for disruptive disorders versus anxiety disorders on the CBCL Attention Problems $(F=4.1, p<.05)$, Delinquent Behavior $(F=4.8, p<.05)$, and Aggressive Behavior scales $(F=7.4, p<.01)$.

\section{Discussion}

The present study's findings may have been influenced by selection factors associated with referral, resulting in an inflated rate of comorbidity in a referred sample like the one we used. Therefore, the results should be interpreted with caution. However, by using a clinical sample instead of a community sample, we were able to conduct more rigorous, focused comparisons for disorders that may be too infrequent in a community sample to be feasible.

\section{CBCL Total Problem Score}

Despite a considerable overlap between caseness defined by the CBCL total problem score and caseness defined by DSM-III-R criteria, there also was a divergence between both approaches. Of the sample, $63 \%$ fulfilled criteria for a DSM-III-R disorder, whereas $47 \%$ scored in the clinical range of the CBCL total problem score. Of the 146 subjects with a DSM-III-R diagnosis, $86(59 \%)$ also had a CBCL total problem score in the clinical range. Conversely, of the 109 subjects with a CBCL total problem score in the clinical range, $79 \%$ also met criteria for a DSM-III-R diagnosis. This disparity between subjects who could be regarded as deviant on the basis of the two different morbidity criteria (CBCL Total 
Problem score in the clinical range versus DSM-III-R criteria) may have been caused by the fact that the total problem score of the CBCL reflects a broad range of problems reflecting overall dysfunctioning of the individual, whereas subjects who fulfil DSM-III-R criteria for a disorder may have an isolated problem without necessarily showing overall dysfunctioning.

In order to test this assumption, we compared the mean number of DISC diagnoses for subjects whose CBCL Total Problem scores were in the clinical range (2.42) versus the mean number of DISC diagnoses for subjects whose CBCL total problem scores were below the CBCL clinical threshold (1.76). This difference was significant (Wilcoxon Rank Sum $W$ test; $Z=2.89 ; p<.01$ ).

Many studies have used the total problem score of the CBCL for screening purposes in community surveys, presuming a relation between the presence of DSM-III-R disorders and the CBCL total problem score (see Verhulst, 1995a, b). In the present study, we demonstrated significant relations between CBCL Total Problem scores in the clinical range and most DSM-III-R disorders (except Separation Anxiety Disorder), which supports the use of the Total Problem score of the CBCL for screening purposes.

\section{CBCL Syndrome Scale Scores}

Withdrawn. Logistic regression analyses indicated that scores in the clinical range of the CBCL Withdrawn scale predicted the following DSM-III-R diagnoses, independently of other scales: Generalized Anxiety Disorder, Major Depression, Dysthymia, and any mood disorder. Scores in the clinical range of the Withdrawn scale were indicative of a broad range of DSM-III-R disorders, rather than one specific diagnosis.

The CBCL scale Withdrawn contains the items (in abbreviated form): Would rather be alone; Refuses to talk; Secretive: Shy or timid; Stares blankly; Sulks; Underactive; Unhappy, sad, depressed; Withdrawn. If we examine the content of the CBCL Withdrawn scale, we can conclude that this scale shows resemblance with DSM-III-R Avoidant Disorder of Childhood or Adolescence. However, the frequency of Avoidant Disorder $(3 \%)$ was smaller than the frequency of scores in the clinical range of the CBCL scale Withdrawn (9\%). Most subjects who scored in the clinical range of the $\mathrm{CBCL}$ scale Withdrawn did not fulfil the criteria for Avoidant Disorder.

DSM-IV (American Psychiatric Association, 1994) has excluded the diagnosis of Avoidant Disorder of Childhood and Adolescence from the list of disorders usually first evident in childhood or adolescence. DSM-IV lacks the possibility of diagnosing problems representing social withdrawal without anxiety symptoms in children and adolescents other than those shown by children with pervasive developmental disorders. Even in the absence of a clear DSM-IV counterpart for the CBCL scale Withdrawn for children and adolescents, the present study has demonstrated a clear correspondence between this scale and diagnostic categories of major anxiety and mood disorders. Despite the lack of specificity, it may well be that high scores on the CBCL scale Withdrawn represent an important problem area usually concomitant with anxiety or mood disorders that deserve attention and which may be a focus of treatment irrespective of the main diagnosis.

Somatic Complaints. Scores in the clinical range of the Somatic Complaints scale predicted, independently of other scales, the DSM-III-R categories: Overanxious Disorder, any anxiety disorder, Major Depression, Dysthymia, and ADHD. The highest odds ratio (11.1) was between the Somatic Complaints scale and Overanxious Disorder. The DSM-III-R category Overanxious Disorder contains criteria concerning somatic complaints such as headaches and stomach-aches for which no physical basis can be established. The type of problems as listed in DSM-III-R are similar to items comprising the CBCL Somatic Complaints scale. The criteria for the DSM-III-R category of Generalized Anxiety Disorder, with which the CBCL scale Somatic Complaints did not have a significant relationship, contains physical symptoms that are related to motor tension and autonomic hyperactivity. The nature of these physical symptoms is different from the physical symptoms comprising the DSM-III-R diagnosis of Overanxious Disorder as well as the problems comprising the CBCL scale Somatic Complaints.

The relationship between the Somatic Complaints scale and the DSM-III-R mood disorders reflects the fact that depressive disorders are often accompanied by somatic complaints. McCauley, Carlson, and Calderon (1991), for instance, reported that $70 \%$ of children with a diagnosis of depression had significant somatic complaints.

Anxious/Depressed. Scores on the CBCL scale Anxious/Depressed in the clinical range predicted, independently of other CBCL scales, the majority of DSM-III-R anxiety and mood disorders. By far the strongest relation of the Anxious/Depressed scale was with the DSM-III-R category Generalized Anxiety Disorder, and to a somewhat lesser extent with Overanxious Disorder. The significant association between the Anxious/Depressed scale and any disruptive DSM-III-R disorder corroborates with the results of other studies showing high rates of comorbidity between anxiety disorders and disruptive behaviors (e.g. Last, Perrin, Hersen, \& Kazdin, 1992; Last, Strauss, \& Francis, 1987), and between depression and disruptive behaviors (Harrington, 1993).

Social Problems. The CBCL Social Problems scale, which includes items such as Acts too young; Too dependent; Does not get along with peers; Gets teased; Not liked by peers; Clumsy; and Prefers younger kids, showed a weak, though significant and rather specific relation with Oppositional Defiant Disorder. This indicates that the CBCL Social Problems scale does not have a strong counterpart in DSM nosology, but represents an area of problematic functioning in interpersonal relationships that may complicate oppositional behaviors.

Thought Problems. Scores in the clinical range on the CBCL scale Thought Problems were not predictive of any DSM-III-R diagnosis independent of other CBCL scales. However, if we included scores just below the clinical threshold but higher than a $T$ score of 67 (this scoring range was named the borderline clinical range by 
Achenbach, 1991a), the Thought Problems scale predicted Simple Phobia, Social Phobia, Separation Anxiety Disorder, any mood disorder, and a positive psychosis screen, independently of other CBCL scales, with the highest odds ratio of 26.7 for the prediction of a positive psychosis screen. These findings indicated that the scale may be useful to detect psychotic disorders, but lacks specificity.

Attention Problems. Scores in the clinical range of the Attention Problems scale predicted, independently of other scales, the following DSM-III-R diagnoses: Attention-deficit Hyperactivity Disorder with or without comorbidity, Oppositional Defiant Disorder, and any disruptive disorder without comorbidity. The finding that the Attention Problems scale was the only significant predictor of "pure" Attention-deficit Hyperactivity Disorder confirmed that this scale was able specifically to assess problems of attention and hyperactivity as captured by DSM-III-R nosology. This supported the finding of Biederman et al. (1993), who reported that scores above $T 60$ of the 1991-CBCL Attention Problems scale strongly predicted DSM-III-R ADHD. The association between the CBCL Attention Problems scale with Oppositional Defiant Disorder reflects the oftenreported comorbidity between hyperactivity and aggressive behaviors.

Aggressive Behavior. The clinical threshold of the Aggressive Behavior scale predicted, independently of other CBCL scales, the following DSM-III-R diagnoses: Attention Deficit Disorder, Oppositional Defiant Disorder, Conduct Disorder, and any disruptive behavior. This indicated that scores in the clinical range of the Aggressive Behavior scale predominantly assessed disruptive or externalizing behaviors.

The highest odds ratio (37.9) was for Oppositional Defiant Disorder. However, scores on the Aggressive Behavior scale in the clinical range were also indicative of ADHD and Conduct Disorder, which reflected the great extent of comorbidity between disruptive disorders.

Delinquent Behavior. Scores on the Delinquent Behavior scale in the clinical range predicted, independently of other CBCL scales, the following DSM-III-R diagnoses: any anxiety disorder, Dysthymia, any mood disorder, Oppositional Defiant Disorder, Conduct Disorder, and any disruptive disorder. Despite the wide range of DSM-III-R diagnoses associated with the Delinquent Behavior scale, this scale was very specifically associated with the DSM-III-R diagnosis of Conduct Disorder, because the odds ratio of 71.9 was the highest odds ratio that we found between any $\mathrm{CBCL}$ syndrome scale and DSM-III-R diagnosis in our study.

Scores on the Delinquent Behavior scale predicted the DSM-III-R diagnosis of Dysthymia. As we have shown, scores on the CBCL Aggressive Behavior scale predicted Major Depression independently of other CBCL scales. This indicates a link between depressive symptoms with $\geqslant 6$ months duration as listed in the DSM-III-R, and aggressive behavior as tapped by the CBCL Aggressive Behavior syndrome, which reflects problem behavior that is usually not likely to cause trouble with the law (e.g. argues, brags, mean to others, demands attention, destroys own things). Conversely, the DSM-III-R diagnosis of Dysthymia, which is comprised of depressive symptoms of $\geqslant 1$ years duration, was predicted by the Delinquent Behavior scale. The CBCL Delinquent Behavior scale comprises behaviors that might cause trouble with the law (e.g. sets fires, steals, and vandalism).

The boundaries between the DSM-III-R diagnoses of Major Depression and Dysthymia are unclear, especially in children and adolescents. The major distinction between both DSM-III-R diagnoses seems to be the duration of the disturbance in mood. The fact that we found an association between the CBCL syndrome Aggressive Behavior with Major Depression and the CBCL syndrome Delinquent Behavior with Dysthymia suggests that subjects who had a more chronic mood disturbance were at higher risk of showing delinquent behaviors.

\section{Conclusions}

Logistic regression analyses, testing the association between each CBCL scale and each DSM-III-R diagnosis separately, demonstrated numerous significant associations between both sets of diagnostic constructs, except for the DSM-III-R diagnosis Social Phobia, which was only predicted by the CBCL Withdrawn scale, and the DSM-III-R diagnosis Separation Anxiety Disorder, which was only predicted by the CBCL Withdrawn and Anxious/Depressed scales. From the broad range of associations between CBCL scales and various DSM-III-R diagnoses, one should not hastily conclude that this is due to the inability of the CBCL scales to detect "clearcut diagnostic constructs" as derived from the DISC clinical interview. The broad range of DSM-III-R diagnoses associated with CBCL scales is largely affected by the high level of comorbidity, which is an intrinsic feature of psychopathology in children and adolescents. Usually, adults tend to overfocus on problem behaviors that have the greatest impact on the environment, and tend to overlook other areas of malfunctioning that may also be important but that are not a nuisance to the environment. Our study showed the high level of comorbidity in a patient sample, with individuals who may be clinically deviant in several different areas of functioning without implying the existence of separate disorders.

In the multivariate logistic regression analyses, we controlled for the interrelatedness among the $\mathrm{CBCL}$ scales (but not among the DISC-derived DSM-III-R diagnoses), and tested the pure contribution of each scale to predict DSM-III-R diagnoses independently of other CBCL scales. Taking account of the interrelatedness among CBCL scales in this way, a different picture emerged, with much more specific and clearcut relations between CBCL scales and DSM-III-R diagnoses. Nevertheless, most DSM-III-R diagnoses were predicted by more than one CBCL scale independent from other scales. These findings are discussed in the light of comorbidity among diagnoses.

Our findings show that the clinical-diagnostic and empirical-quantitative approaches do not converge to a degree that one approach can replace the other. Instead of discarding one for the other, we hold the point of view that both approaches are needed, because the combination can aid in increasing our knowledge of psy- 
chopathology in children and adolescents. For example, our findings demonstrated that for the CBCL syndromes Withdrawn and Social Problems there was no clear counterpart in DSM nosology. However, if we know that an individual has significant problems in these areas of functioning, this information may be an important addition to a clinical diagnosis. It is an empirical question as regards which approach has the greatest power in terms of long-term prognosis and effect of treatment strategy.

Acknowledgements - The authors wish to thank their colleagues of RIAGG RNO for their kind cooperation, and are grateful to Ester de Kleijn, Mary Cheung, Claudia Stigter, Judit Wesseling, Francine van der Velden, Hester Ponte, and David Stevens for performing the DISC interviews.

\section{References}

Achenbach, T.M. (1991a). Integrative guide to the 1991 CBCL/4-18, YSR, and TRF profiles. Burlington, VT: University of Vermont Department of Psychiatry.

Achenbach, T. M. (1991b). Manual for the Child Behavior Checklist/4-18 and 1991 profile. Burlington, VT: University of Vermont Department of Psychiatry.

Achenbach, T. M., \& Edelbrock, C. S. (1983). Manual for the revised Child Behavior Checklist and profile. Burlington, VT: University Associates in Psychiatry.

American Psychiatric Association. (1980). Diagnostic and statistical manual of mental disorders (3rd edn). Washington, DC: Author.

American Psychiatric Association. (1987). Diagnostic and statistical manual of mental disorders (3rd edn-revised). Washington, DC: Author.

American Psychiatric Association. (1994). Diagnostic and statistical manual of mental disorders (4th edn). Washington, DC: Author.

Biederman, J., Faraone, S. V., Doyle, A., Krifcher Lehman, B., Kraus, I., Perrin, J., \& Tsuang, M. T. (1993). Convergence of the Child Behavior Checklist with structured interview-based psychiatric diagnoses of ADHD with and without comorbidity. Journal of Child Psychology and Psychiatry, 34, 1241-1251.

Edelbrock, G., \& Costello, A. J. (1988). Convergence between statistically derived behavior problem syndromes and child psychiatric diagnoses. Journal of Abnormal Child Psychology, 16, 219-231.

Gould, M. S., Bird, H., \& Staghezza Jaramillo, B. (1993). Correspondence between statistically derived problem syndromes and child psychiatric diagnoses in a community sample. Journal of Abnormal Child Psychology, 21, 287-313.

Harrington, R. (1993). Depressive disorder in childhood and adolescence. New York: Wiley.

Jensen, P. S., Salzberg, A. D., Richters, J. E., \& Watanabe, H. K. (1993). Scales, diagnoses and child psychopathology.
I. CBCL and DISC relationships. Journal of the American Academy of Child and Adolescent Psychiatry, 32, 397-406.

Last, C. G., Perrin, S., Hersen, M., \& Kazdin, A. E. (1992). DSM-III-R anxiety disorders in children : Sociodemographic and clinical characteristics. Journal of the American Academy of Child and Adolescent Psychiatry, 31, 1070-1076.

Last, C. G., Strauss, C. G., \& Francis, G. (1987). Comorbidity among childhood anxiety disorders. Journal of Nervous and Mental Disease, 175, 726-730.

McCauley, E., Carlson, G. A., \& Calderon, R. (1991). The role of somatic complaints in the diagnosis of depression in children and adolescents. Journal of the American Academy of Child and Adolescent Psychiatry, 30, 631-635.

NIMH (National Institute of Mental Health). (1992). Diagnostic interview schedule for children. New York: New York State Psychiatric Institute Division of Child and Adolescent Psychiatry.

Piacentini, J., Shaffer, D., Fischer, P., Schwab-Stone, M., Davies, M., \& Giola, P. (1993). The Diagnostic Interview Schedule for Children-revised version (DISC-R). III. Concurrent criterion validity. Journal of the American Academy of Child and Adolescent Psychiatry, 32, 658-665.

Schwab-Stone, M., Fisher, P., Piacentini, J., Shaffer, D., Davies, M., \& Briggs, M. (1993). The Diagnostic Interview Schedule for Children-revised version (DISC-R), II. Test-retest reliability. Journal of the American Academy of Child and Adolescent Psychiatry, 32, 651-657.

Shaffer, D., Schwab-Stone, M., Fisher, P., Cohen, P., Piancentini, J., Davies, M., Conners, C. K., \& Regier, D. (1993). The Diagnostic Interview Schedule for Childrenrevised version (DISC-R). I. Preparation, field testing, interrater reliability, and acceptability. Journal of the American Academy of Child and Adolescent Psychiatry, 32, 643-650.

Steingard, R., Biederman, J., Doyle, A., \& Sprich-Buckminster, S. (1992). Psychiatric comorbidity in attention deficit disorder: Impact on the interpretation of Child Behavior Checklist results. Journal of the American Academy of Child and Adolescent Psychiatry, 31, 449-461.

Van Westerlaak, J. H., Kropman, J. A., \& Collaris, J. W. M. (1975). Beroepenklapper. Nijmegen, The Netherlands: Instituut voor toegepaste sociologie.

Verhulst, F. C. (1995a). The epidemiology of child and adolescent psychopathology: Strengths and limitations. In F. C. Verhulst \& H. M. Koot (Eds.), The epidemiology of child and adolescent psychopathology (pp. 1-21). London: Oxford University Press.

Verhulst, F. C. (1995b). A review of community studies. In F. C. Verhulst \& H. M. Koot (Eds.), The epidemiology of child and adolescent psychopathology (pp. 146-177). London: Oxford University Press.

Verhulst, F. C., \& Achenbach, T. M. (1995). Empirically based assessment and taxonomy of psychopathology: Cross-cultural applications. European Child and Adolescent Psychiatry, 4, 61-76.

Verhulst, F. C., Berden, G. F. M. G., \& Sanders-Woudstra, J. A. R. (1985). Mental health in Dutch children. II. The prevalence of psychiatric disorder and relationship between measures. Acta Psychiatrica Scandinavica, 72 (Suppl. 342).

Accepted manuscript received 10 October 1996 
This document is a scanned copy of a printed document. No warranty is given about the accuracy of the copy. Users should refer to the original published version of the material. 